\title{
Inhibition of the Replication of Feline Immunodeficiency Virus by Lentiviral Vector- Mediated RNA Interference in Feline Cell Lines
}

\author{
Kenji BABA ${ }^{1) *}$, Yuko GOTO-KOSHINO ${ }^{1)}$, Fuminori MIZUKOSHI ${ }^{1)}$, Asuka SETOGUCHI-MUKAI ${ }^{1) *}$, \\ Yasuhito FUJINO $^{1)}$, Koichi $\mathrm{OHNO}^{1)}$ and Hajime TSUJIMOTO ${ }^{1) * *}$ \\ ${ }^{1)}$ Department of Veterinary Internal Medicine, Graduate School of Agricultural and Life Sciences, The University of Tokyo, 1-1-1 Yayoi, \\ Bunkyo-ku, Tokyo 113-8657, Japan
}

(Received 12 March 2008/Accepted 9 April 2008)

ABSTRACT. RNA interference (RNAi) is a sequence-specific RNA degradation process. To inhibit feline immunodeficiency virus (FIV) replication by RNAi, we generated a lentiviral vector expressing a short hairpin RNA (shRNA) that targeted the gag gene of FIV (shGag). shGag transfer significantly inhibited viral replication in cell lines that were chronically infected with FIV, i.e., the 3201/UK8 ${ }^{\text {low }}$, 3201/ $\mathrm{UK} 8^{\text {high }}$, FL4, and CRFK/FIV cell lines. Moreover, 3201 cells were transduced with the lentiviral vectors and then inoculated with FIV. Although the amount of FIV proviral DNA in shGag-transduced cells was similar to that in the cells transduced with unrelated shRNA or mock-transduced cells, the amount of reverse transcriptase (RT) activity was significantly reduced in the culture supernatant of shGagexpressing cells from 15 to 27 days after inoculation. Thirty days after inoculation, no significant difference was observed in the RT activities but virus with a mutation in the target region of shGag was detected in approximately $21 \%$ of the replicated viruses. Therefore, abolishment of the silencing effect of shGag may be due to reasons other than the emergence of escape mutants. These results are useful for developing an RNAi-based gene therapy strategy for controlling FIV infection.

KEY WORDS: feline immunodeficiency virus, gene therapy, lentiviral vector, short hairpin RNA.

Feline immunodeficiency virus (FIV) belongs to the genus Lentivirus of the family Retroviridae that includes human immunodeficiency virus-1 (HIV-1). FIV infection induces acquired immunodeficiency syndrome (AIDS) by the progressive depletion of CD4-positive $\mathrm{T}$ cells in infected cats, a scenario similar to that of HIV-1 infection in humans $[13,25]$. Since no effective antiviral therapy has been established, only supportive therapy is carried out for FIVinfected cats. Although some reverse transcriptase (RT) inhibitors and protease inhibitors have been shown to improve the clinical symptoms in cats that were naturally or experimentally infected with FIV $[8,15]$, these drugs are not used therapeutically in clinical settings because of their toxicities and inadequate effects $[1,12]$. Therefore, other therapeutic strategies are required for controlling FIV infection in cats.

RNA interference (RNAi) is a process of sequence-specific RNA degradation that is conserved in eukaryotes [9, 28]. At present, RNAi technology is regarded as a possible gene therapy strategy for treating various diseases because it

\footnotetext{
* CuRRent ADDRESS: BABA, K., Laboratory of Viral Pathogenesis, Center for Emerging Virus Research, Institute for Virus Research, Kyoto University, 53 Shogoin-Kawaracho, Sakyo-ku, Kyoto 606-8507, Japan.

Setoguchi-Mukai, A., Laboratory of Veterinary Internal Medicine, Faculty of Agriculture, Kagoshima University, 1-21-24 Korimoto, Kagoshima 890-0065, Japan.

**Correspondence to: Tsujimoto, H., Department of Veterinary Internal Medicine, Graduate School of Agricultural and Life Sciences, The University of Tokyo, 1-1-1 Yayoi, Bunkyo-ku, Tokyo 113-8657, Japan.

e-mail: atsuji@mail.ecc.u-tokyo.ac.jp
}

can be used to strongly inhibit the expression of specific molecules. Recent studies have demonstrated that RNAi can target a variety of human viruses $[10,11,17-19,21,26$, 29, 31]. Therefore, RNAi technology may possibly be used as a therapeutic strategy for controlling specific pathogens. Previously, we reported that chemically synthesized small interfering RNAs (siRNAs) and a retroviral vector-mediated short hairpin RNA (shRNA) homologous to FIV gag gene efficiently inhibited the replication of FIV by the degradation of viral RNA in chronically FIV-infected cell lines [2]. However, there are problems to use these delivery methods for clinical practices. Most important problems of siRNA are the low efficiency of transfection in primary cells and its transient silencing effect. Although the transduction of shRNA by retroviral vector can induce the inhibitory effect persistently, there is the limitation of transduction into nondividing cells. On the other hand, lentiviral vector can achieve efficient transduction into various cells including non-dividing cells. Therefore, lentiviral vector is considered as the most suitable delivery method to use RNAi technology as gene therapy strategy.

In this study, we generated an shRNA-expressing lentiviral vector that targeted the gag gene of FIV, and the resulting therapeutic and prophylactic effects were examined in feline cell lines. We demonstrated the inhibitory effect of lentiviral vector-mediated FIV-specific shRNA on FIV replication in both acutely infected cells and cells with established infection. These results provide useful information for developing an RNAi-based gene therapy strategy for controlling FIV infection. 


\section{MATERIALS AND METHODS}

Virus: A field isolate of FIV, i.e., the UK8 strain [27], was kindly provided by Dr. Janet K. Yamamoto (University of Florida, Gainesville, FL). The culture supernatant of a feline T-lymphoid cell line 3201 [30] infected with the FIV UK8 strain was harvested and stored at $-80^{\circ} \mathrm{C}$ for subsequent use. Aliquots of the same viral stock were used in all experiments.

Cells: Four feline T-lymphoid cell lines, i.e., 3201, 3201/ $\mathrm{UK} 8^{\text {low }}, 3201 / \mathrm{UK} 8^{\text {high }}$, and FL4 [32], were maintained in RPMI 1640 medium (Sigma, St. Louis, MO) supplemented with $10 \%$ heat-inactivated fetal calf serum (FCS) and penicillin/streptomycin. Two cell lines chronically infected with the FIV UK8 strain, i.e., the $3201 / \mathrm{UK} 8^{\text {high }}$ and $3201 / \mathrm{UK} 8^{\text {low }}$ cell lines, were originally generated in our laboratory, with the former producing more virus than the latter. A feline fibroblastic cell line (CRFK) [7] that was chronically infected with the FIV Petaluma strain (CRFK/FIV) was maintained in Dulbecco's modified Eagle medium (DMEM) (Sigma) containing 10\% heat-inactivated FCS and penicillin/streptomycin. HEK293T cells (ATCC CRL11268) were maintained in DMEM supplemented with $10 \%$ heat-inactivated FCS, penicillin/streptomycin, $2 \mathrm{mM}$ Lglutamine, and $1 \mathrm{mM}$ sodium pyruvate.

Design of shRNAs: The shRNA sequence specific to the gag gene of FIV (shGag) was designed on the basis of an siRNA sequence that efficiently inhibited FIV replication in CRFK/FIV cells, as reported earlier [2]. A scrambled shGag sequence, which was nonhomologous to FIV, was used as a control (shScr). These shRNA oligonucleotides were designed to contain a sense strand with a 19-nucleotide sequence followed by a short spacer (AGGATC), a reverse complement of the sense strand, and six thymidines as the RNA polymerase III transcriptional stop signal.

Construction of lentiviral vectors: The HIV-1-derived shRNA-expressing lentiviral vectors were generated by the system originally established by Sam Wilson et al. (University College London, London, UK; unpublished data). First, shRNA oligonucleotides were subcloned into a cloning plasmid pU6-1-linker (a gift from Dr. Sam Wilson) that contained a human U6 RNA polymerase III promoter to drive shRNA expression. The SalI-XbaI fragment from the pU61-linker was ligated with an annealing product of the sense oligonucleotides (5'-TCGAGCCAGAAAGTACCCTAG AAAGGATCTTCTAGGGTACTTTCTGGCTTTTTT-3') (nt 1626-1644 in the FIV14 genome [23], GenBank accession number: M25381) and antisense oligonucleotides (5'CTAGAAAAAAGCCAGAAAGTACCCTAGAAGATCCTTTCTAGGGTACTTTCTGGC-3') to generate pU6-1linker-shGag. pU6-1-linker-shScr was constructed by ligating an annealing product of sense oligonucleotides (5'TCGAGACAAGTAGACACGACTACAGGATCGTAGTCGTGTCTACTTGTCTTTTTT-3') and antisense oligonucleotides (5',CTAGAAAAAAGACAAGTA GACACGACTACGATCCTGTAGTCGTGTCTACTTGTC-3') with the SalI-XbaI fragment from the pU6-1- linker. Second, the shRNA transfer lentiviral vector genome plasmids pSIN-PAC-U6shGag and pSIN-PACU6shScr were generated by subcloning the shRNA expression construct from pU6-1-linker-shGag or pU6-1-linkershScr, respectively, into pSIN-PAC (a gift from Dr. Greg Towers, University College London, London, UK) containing a puromycin-resistant gene expression cassette. All constructs were verified by nucleotide sequencing.

Preparation of lentiviral vectors: pCMVR8.91, a packaging plasmid, and pMD-G, a vesicular stomatitis virus envelope glycoprotein (VSV-G) expression plasmid, were generously provided by Dr. Didier Trono (Salk Institute, La Jolla, CA). VSV-G pseudotyped lentiviral vectors were generated by transient cotransfection of vector genome plasmids pCMVR8.91 and pMD-G into HEK293T cells by means of the cationic lipid complex method (Lipofectamine 2000; Invitrogen, Rockville, MD). The culture supernatants containing the produced viruses were harvested at $48 \mathrm{hr}, 72$ $\mathrm{hr}$, and $96 \mathrm{hr}$ after transfection, and concentrated by centrifugation at $50,000 \mathrm{~g}$ at $4^{\circ} \mathrm{C}$ for $120 \mathrm{~min}$. Aliquots of the concentrated viruses were stored at $-80^{\circ} \mathrm{C}$ for subsequent use.

Transduction of lentiviral vectors: Twelve-well plates were coated with $5 \mu \mathrm{g}$ of Retronectin (TaKaRa Shuzo, Kyoto, Japan) per $\mathrm{cm}^{2}$ and stocked lentivirus. Four cell lines, i.e., 3201, 3201/UK $8^{\text {low }}, 3201 / \mathrm{UK} 8^{\text {high }}$, and FL4 cells $\left(2 \times 10^{5}\right.$ cells per well), were seeded on these wells. The resulting multiplicity of infection (MOI) was calculated to be 5 . After incubation at $37^{\circ} \mathrm{C}$ for $48 \mathrm{hr}$, the cells were washed twice with PBS and then cultured in the presence of $1 \mu \mathrm{g}$ of puromycin per $\mathrm{m} l$ for 10 days to select stably transduced cells. CRFK/FIV cells were inoculated with lentivirus by adding $8 \mu \mathrm{g}$ of polybrene per $\mathrm{ml}$.

FIV infection: Three cell lines, i.e., 3201 and 3201 transduced with either shGag or shScr, were infected with FIV. Cells $\left(2 \times 10^{6}\right.$ cells per $\left.\mathrm{m} l\right)$ were inoculated with a viral stock of the FIV UK8 strain (RT activity equivalent to $100,000 \mathrm{cpm}$ per $\mathrm{ml}$ ) at $37^{\circ} \mathrm{C}$ for $2 \mathrm{hr}$, washed twice with PBS, and resuspended to a final concentration of $4 \times 10^{5}$ cells per $\mathrm{m} l$. Viable cells were counted by trypan blue exclusion every 3 days and passaged to adjust the concentration to $4 \times 10^{5}$ cells per $\mathrm{m} l$. Cell-free culture supernatants were harvested every 3 days from 0 to 33 days after inoculation with FIV to measure the RT activities.

Detection of FIV proviral DNA: High-molecular-weight DNA samples were extracted from cells inoculated with FIV for $2 \mathrm{hr}$ by using the QIAamp DNA Blood Mini Kit (Qiagen, Valencia, CA), according to the manufacturer's protocol. Five hundred nanograms of DNA from each sample was amplified by polymerase chain reaction (PCR) using primer pairs specific for the gag gene of the FIV provirus. As an internal control, the p27kipl gene was also amplified. The sequences of the PG-1/PG-4 [16] and KAS/ KAR [22] primers were as follows: PG-1, 5'-CTAGGAGGTGAGGAAGTTCAACTATG-3' (nt 1120 to 1145 in the FIV14 genome); PG-4, 5'-CTGGTGAGCCTATTTCTTGACAAGC-3' (nt 1681 to 1657 in the FIV14 genome); KAS, 5'-AGGCGGTCGTAGAGACCTG-3' (nt 35 to 53 in 
the feline p27kipl cDNA [22]); and KAR, 5'-TTCCCGCTGACATCCTGG-3' (nt 396 to 379 in the feline p27kipl cDNA). Following initial denaturation at $95^{\circ} \mathrm{C}$ for $5 \mathrm{~min}$, we carried out 29,32 , or 35 cycles of denaturation at $94^{\circ} \mathrm{C}$ for $45 \mathrm{sec}$, annealing at $60^{\circ} \mathrm{C}$ for $45 \mathrm{sec}$, and polymerization at $72^{\circ} \mathrm{C}$ for $45 \mathrm{sec}$; this was followed by a final extension at $72^{\circ} \mathrm{C}$ for $10 \mathrm{~min}$. The PCR products were subjected to electrophoresis in a $2 \%$ agarose gel and visualized by ethidium bromide staining.

Reverse transcriptase activity assay: Ten microliters of the cell-free culture supernatant was added to $50 \mu l$ of RT buffer containing $0.5 \mu \mathrm{g}$ of poly(rA) $\cdot \mathrm{p}(\mathrm{dT})_{12-18}$ (Amersham Biosciences, Piscataway, NJ) per $\mathrm{m} l, 0.1 \%$ Nonidet NP-40, $60 \mathrm{mM}$ Tris- $\mathrm{HCl}, 75 \mathrm{mM} \mathrm{KCl}, 5 \mathrm{mM} \mathrm{MgCl}, 1 \mathrm{mM}$ EDTA, $4 \mathrm{mM}$ DTT, and $10 \mu \mathrm{Ci}$ of $\left[{ }^{32} \mathrm{P}\right] \mathrm{dTTP}$ (Amersham Biosciences) per $\mathrm{ml}$. After incubation at $37^{\circ} \mathrm{C}$ for $2 \mathrm{hr}, 10 \mu \mathrm{l}$ of the mixture was spotted onto DEAE filter paper (Wallac Oy, Turku, Finland $)$ and washed 4 times with $2 \times$ SSC $(1 \times$ SSC is $0.15 \mathrm{M}$ sodium chloride plus $15 \mathrm{mM}$ sodium citrate) and once with $99 \%$ ethanol. The filter papers were then dried, and the incorporated radioactivity on the filter paper was measured by autoradiography.

Sequencing analysis: Virion-associated RNA was extracted from cell-free culture supernatants 30 days after FIV inoculation by using a QIAamp viral RNA kit (QIAGEN). cDNA samples were synthesized from $0.5 \mu \mathrm{g}$ of viral RNA with the RNA PCR kit (Applied Biosystems, Foster City, CA). Using the GAGS (5'-ATGCAGTGTAGAGCATGGTA-3', nt 1387 to 1406 in the FIV14 genome) and GAGR (5'-TTGCTGCATTTGATTCACTG-3', nt 1929 to 1910 in the FIV14 genome) primer pair, the FIV gag gene was amplified from viral cDNAs using the Ex Taq DNA polymerase (TaKaRa) according to the manufacturer's instructions. The PCR amplifications consisted of predenaturation $\left(94^{\circ} \mathrm{C}, 2 \mathrm{~min}\right)$ and 35 cycles of denaturation $\left(94^{\circ} \mathrm{C}, 30 \mathrm{sec}\right)$, annealing $\left(60^{\circ} \mathrm{C}, 30 \mathrm{sec}\right)$, and extension $\left(72^{\circ} \mathrm{C}, 45 \mathrm{sec}\right)$; this was followed by a final extension $\left(72^{\circ} \mathrm{C}\right.$, $7 \mathrm{~min}$ ). The 543-bp PCR products were cloned into a plasmid vector (pGEM-T easy vector; Promega, Madison, WI) followed by transformation into competent Escherichia coli cells. The cloned PCR products were sequenced by the dideoxy chain termination method (Applied Biosystems).

Statistical analysis: One-way analysis of variance (ANOVA) was used to test for significant differences in the RT activities of the culture supernatants. If a significant difference was detected by ANOVA, Tukey-Kramer's Honestly Significant Difference test was performed to determine the pair that showed the difference. Statistical significance was defined as $P<0.05$.

\section{RESULTS}

Therapeutic effect of lentiviral vector-mediated shRNA transfer on FIV replication: To determine the therapeutic effect of lentiviral vector-mediated shRNA transfer on FIV replication, we examined the ability of shRNA that targets FIV to inhibit viral replication in chronically FIV-infected
3201/UK8 $8^{\text {low }}, 3201 / \mathrm{UK} 8^{\text {high }}$, FL4, and CRFK/FIV cell lines. The RT activity in the culture supernatants of cells expressing shGag was significantly lower than that of untreated cells whereas that of cells expressing shScr was unaltered (Fig. 1). Similar RT activity reduction was observed in these cell lines regardless of the amount of viral production (78\%, 75\%, 92\%, and $82 \%$ reduction in $3201 / \mathrm{UK} 8^{\text {low }}, 3201 /$ UK ${ }^{\text {high }}$, FL4, and CRFK/FIV, respectively).

Prophylactic effect of lentiviral vector-mediated shRNA transfer on FIV replication: To determine the prophylactic effect of lentiviral vector-mediated shRNA transfer on FIV replication, 3201 cells expressing shGag or shScr were inoculated with FIV. Two hours after inoculation, 29, 32, and 35 cycles of PCR amplification were performed to detect FIV proviral DNA in cells expressing no shRNA and cells expressing shScr or shGag (Fig. 2). FIV proviral DNA was similarly detected in the cells transduced with shGag, unrelated shRNA or no shRNA. The p27kip1 gene was amplified to an equal extent in each cell type. This result demonstrated that in our shRNA transfer system, the incoming genomic RNA of FIV was not efficiently degraded by shRNA that targets the gag gene of FIV.

Next, the inhibitory effect on the late stage of the viral replication cycle was assessed by measuring the RT activities in the culture supernatants. The RT activities in the culture supernatants of these cells were monitored for up to 33 days after inoculation. The RT activity in the culture supernatant of cells expressing shScr or no shRNA was considerably increased from 15 days after inoculation (Fig. 3A). In the cells expressing shGag, the RT activity in the culture supernatant was significantly lower than that of cells expressing shScr or no shRNA from 15 to 27 days after inoculation, although the activity per se gradually increased during this period (Fig. 3A). Massive cell death was observed in the cells expressing shScr or no shRNA from 15 to 18 days after inoculation; this was followed by the recovery of cell viability from 21 days after inoculation (Fig. 3B). In contrast, the viability of shGag-expressing cells was slightly decreased from 21 to 24 days after inoculation (Fig. $3 \mathrm{~B})$. These results demonstrated that lentiviral delivery of shRNA that targets the gag gene of FIV could efficiently delay FIV replication and protect T-lymphoid cells from FIV-induced cell death.

Emergence of virus escaping the shRNA silencing effect: To determine whether replicated viruses had mutations in the target region of shGag, we sequenced the cDNAs cloned from virion-associated RNA in the culture supernatants at 30 days after inoculation. As shown in Table 1, a mutation (1638C to T) in the target region of shGag was detected in 7 of 33 clones $(21 \%)$ from the culture supernatants of cells transduced with shGag but not in those of cells expressing shScr and no shRNA.

\section{DISCUSSION}

RNAi technology is regarded as a new potential therapeutic strategy for treating viral infections in humans. The 

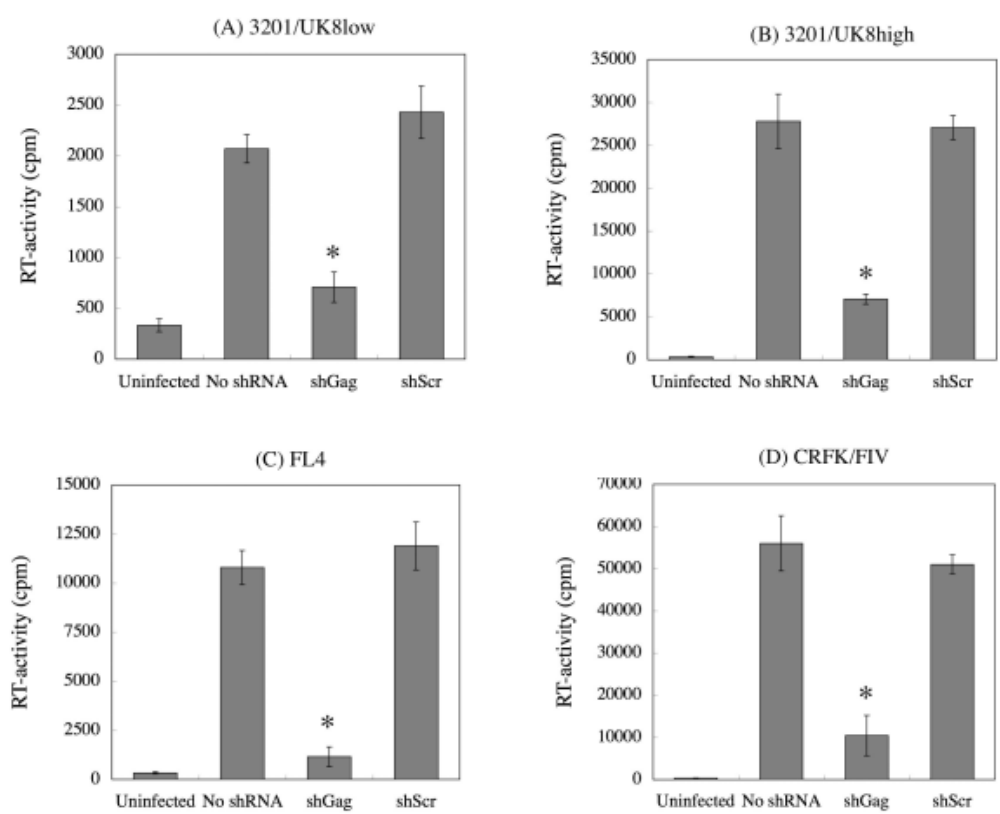

Fig. 1. The therapeutic effect of lentiviral-mediated shRNA transfer on FIV replication in cells with established infection. Four chronically FIV-infected cell lines, i.e., 3201/UK $8^{\text {low }}, 3201 / \mathrm{UK} 8^{\text {high }}$, FL4, and CRFK/FIV, were transduced with shGag- or shScr-expressing lentiviral vector and then cultured for 10 days in the presence of puromycin to select cell lines expressing shGag or shScr. The RT activities in the culture supernatants of $3201 / \mathrm{UK} 8^{\text {low }}(\mathrm{A}), 3201 / \mathrm{UK} 8^{\text {high }}(\mathrm{B})$, FL4 (C), and CRFK/ FIV (D) were measured. The columns and bars show the means and standard deviations, respectively, obtained from the data of triplicate samples. The asterisk indicates statistically significant differences between cells expressing shGag and the controls (shScr and no shRNA).

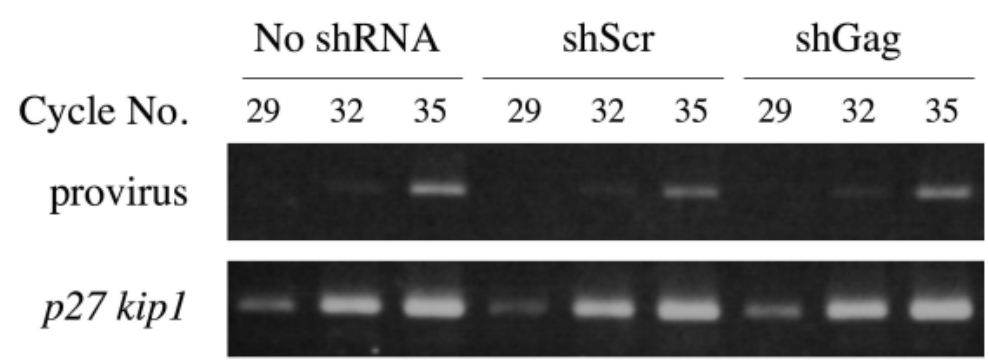

Fig. 2. The effect of lentiviral vector-mediated shRNA transfer on the integration of FIV proviral DNA. Cells expressing shGag, shScr, or no shRNA were inoculated with FIV. Two hours after inoculation, genomic DNA was extracted from each cell and subjected to PCR amplification for FIV proviral DNA with the p27kip1 gene as an internal control. The PCR products after 29, 32, and 35 cycles of amplification were analyzed by agarose gel electrophoresis. A representative result from three independent experiments is shown.

present study was carried out to investigate the possibility of using RNAi technology for controlling FIV infection in cats. We developed a lentiviral vector expressing shRNA that was specific to FIV and examined the prophylactic and therapeutic effects of lentiviral vector-mediated shRNA transfer in feline cell lines. To the best of our knowledge, this is the first report to demonstrate that lentiviral delivery of shRNA can inhibit FIV replication.

Lentiviral delivery of shGag efficiently inhibited FIV replication in cell lines with established FIV infection, thereby demonstrating the therapeutic effect of shRNA that targets FIV (Fig. 1). Surprisingly, similar reduction in RT activity was observed in these cell lines regardless of the amount of viral production. In the cells that FIV actively 
(A)

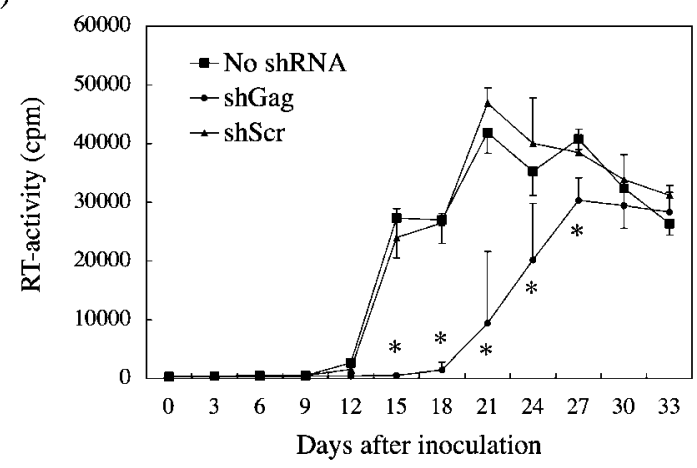

(B)

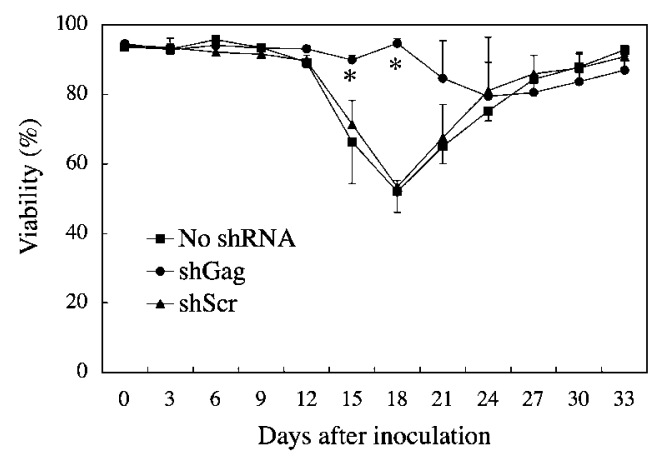

Fig. 3. The prophylactic effect of lentiviral-mediated shRNA transfer on FIV replication. Cells expressing shGag, shScr, or no shRNA were inoculated with FIV and then cultured for up to 33 days after inoculation. (A) The RT activities in the culture supernatants of the cells expressing no shRNA (squares), shScr (triangles), and shGag (circles) were measured every 3 days from 0 to 33 days after inoculation. (B) The viabilities of cells expressing no shRNA (squares), shScr (triangles), and shGag (circles) were calculated by the trypan blue exclusion assay every 3 days from 0 to 33 days after inoculation. Means and standard deviations obtained from the data of triplicate samples are shown. The asterisk indicates statistically significant differences between cells expressing shGag and the controls (shScr and no shRNA).

Table 1. Sequence analysis of the target region of shGag in viral cDNAs cloned from virion-associated RNA in the culture supernatants

\begin{tabular}{cccl}
\hline shRNA & $\mathrm{n}$ & Wild type & Mutated \\
\hline none & 33 & 33 & 0 \\
shScr & 33 & 33 & 0 \\
shGag & 33 & 26 & $7(\mathrm{C} 1638 \mathrm{~T})$ \\
\hline
\end{tabular}

replicates, shRNA transduced with lentiviral vector may also have a tendency to be actively transcribed although the amount of shRNA transcripts in these cell lines were not assessed. In either case, shGag could not completely inhibit viral replication. To achieve a stronger silencing effect, it may be necessary to increase shRNA expression, change the target sequence of shRNA, or target multiple different genes. Targeting different regions of the viral genome may also be effective in reducing the probability of emerging mutant viruses escaping shRNA-mediated degradation.

In this study, inhibition of FIV proviral DNA synthesis was not observed in cells expressing shRNA that targets the gag gene of FIV (Fig. 2). Several reports have demonstrated that the incoming genomic RNA of HIV-1 could be targeted by chemically synthesized siRNA prior to proviral DNA synthesis $[5,6,17]$. On the other hand, siRNA or shRNA is reported to have no effect on the degradation of incoming genomic RNA [14, 20]. The difference in the intracellular localization of chemically synthesized siRNA and shRNA transcribed from the RNA polymerase III promoter may possibly explain this observation. shRNA transcribed from the RNA polymerase III promoter was shown to be mainly localized in the nucleus [24], whereas chemically synthesized siRNA was localized in the cytoplasm [33]. Therefore, chemically synthesized siRNA but not shRNA transcribed from the RNA polymerase III promoter may access incoming viral genomic RNA in the cytoplasm prior to reverse transcription. Alternatively, the site targeted by shGag in this study might be less accessible in viral genomic RNA than in mRNA transcribed from integrated provirus. It is possible that the secondary structure of viral genomic RNA or the binding of viral proteins to genomic RNA limits the access of shRNA to viral genomic RNA.

Although the amount of FIV proviral DNA did not differ considerably among the cells expressing shGag, shScr, and no shRNA, the RT activity in the culture supernatant of cells expressing shGag was significantly lower than that of cells expressing no shRNA or shScr up to 27 days after inoculation (Fig. 3A). These results demonstrate that lentiviral vector-mediated shRNA transfer can inhibit FIV replication in feline T-lymphoid cells by post-transcriptional gene silencing. In addition, FIV-induced cell death was also significantly inhibited in shGag-expressing cells, suggesting that shRNA targets FIV to protect lymphocytes from apoptosis in FIV-infected cats (Fig. 3B). However, slight viral replication and cell death were noticed in the cells expressing shGag at 21 days after inoculation. Twenty-one days after inoculation, the amount of viral transcription might have increased immensely and may have overcome shGag-mediated degradation of target viral RNA. Another possibility is that the mutant virus with mutations in or near the target region might emerge in shGag-expressing cells. However, this is debatable since the mutant virus was not dominant in the culture supernatant of shGag-expressing cells (Table 1). Slight HIV-1 replication, which was not due to the emergence of escaped mutant virus, has been reported in MT4 cells transduced with a lentiviral vector expressing shRNA that targets the vif gene of HIV-1 [20]. Recently, it has been reported that the Tat protein of HIV-1 functions as a suppressor of RNA silencing by interfering with the ability of Dicer to process shRNAs into siRNAs [4]. Further, several RNA or DNA viruses have been shown to develop resistance to RNAi in mammalian cells by producing suppressor viral proteins or RNAs [34]. In FIV, it is more likely that 
viral mechanisms that allow target RNA to escape shRNAmediated degradation are present rather than the emergence of resistant virus through mutations in or near the target sequence.

The goal of gene therapy in the control of FIV infections is to obtain T-lymphocytes that are resistant to FIV. The candidate target cells in RNAi gene therapy for controlling FIV infection are hematopoietic stem cells (HSCs). In theory, the development of a complete hematopoietic system resistant to FIV infection is possible through the introduction of shRNA that targets FIV into pluripotent HSCs because the capacity of self-renewal of these HSCs, as well as their proliferation and differentiation into the lymphoid lineage, is well known. Banerjea et al. demonstrated that HSCs transduced with a lentiviral vector expressing siRNA that targets the HIV-1 rev gene were allowed to mature into T-lymphocytes in vivo in SCID-hu mouse thy/liv grafts, and these HSC-derived siRNA-expressing T cells and macrophages showed marked resistance to HIV-1 infection [3]. Although systems for the isolation and purification of feline HSCs have not been established, in future, lentiviral vectormediated transfer of FIV-specific shRNA into HSCs will be a useful gene therapy strategy for controlling FIV infection in cats.

ACKNOWLEDGMENTS. We would like to thank Dr. Janet K. Yamamoto (University of Florida, Gainesville, FL) for providing a stock of the FIV UK8 strain, Dr. Sam Wilson (University College London, London, UK) for the gift of the pU6-1-linker, Dr. Greg Towers (University College London, London, UK) for the gift of pSIN-PAC, Dr. Didier Trono (Salk Institute, La Jolla, CA) for the gift of pCMVR8.91 and pMD-G, and Dr. Takayuki Miyazawa for supporting this study. This study was supported by grants from the Japan Health Science Foundation and the Ministry of Education, Culture, Sports, Science and Technology, Japan.

\section{REFERENCES}

1. Arai, M., Earl, D. D. and Yamamoto, J. K. 2002. Is AZT/3TC therapy effective against FIV infection or immunopathogenesis? Vet. Immunol. Immunopathol. 85: 189-204.

2. Baba, K., Mizukoshi, F., Goto-Koshino, Y., Setogucji-Mukai, A., Fujino, Y., Ohno, K. and Tsujimoto, H. 2007. Application of RNA interference for inhibiting the replication of feline immunodeficiency virus in chronically infected cell lines. Vet. Microbiol. 120: 207-216.

3. Banerjea, A., Li, M. J., Bauer, G., Remling, L., Lee, N. S., Rossi, J. and Akkina, R. 2003. Inhibition of HIV-1 by lentiviral vector-transduced siRNAs in T lymphocytes differentiated in SCID-hu mice and CD34+ progenitor cell-derived macrophages. Mol. Ther. 8: 62-71.

4. Bennasser, Y., Le, S. Y., Benkirane, M. and Jeang, K. T. 2005. Evidence that HIV-1 encodes an siRNA and a suppressor of RNA silencing. Immunity 22: 607-619.

5. Capodici, J., Kariko, K. and Weissman, D. 2002. Inhibition of HIV-1 infection by small interfering RNA-mediated RNA interference. J. Immunol. 169: 5196-5201.
6. Coburn, G. A. and Cullen, B. R. 2002. Potent and specific inhibition of human immunodeficiency virus type 1 replication by RNA interference. J. Virol. 76: 9225-9231.

7. Crandell, R. A., Fabricant, C. G. and Nelson-Rees, W. A. 1973. Development, characterization, and viral susceptibility of a feline (Felis catus) renal cell line (CRFK). In Vitro 9: 176-185.

8. Egberink, H., Borst, M., Niphuis, H., Balzarini, J., Neu, H., Schellekens, H., De Clercq, E., Horzinek, M. and Koolen, M. 1990. Suppression of feline immunodeficiency virus infection in vivo by 9-(2- phosphonomethoxyethyl)adenine. Proc. Natl. Acad. Sci. U. S. A. 87: 3087-3091.

9. Fire, A., Xu, S., Montgomery, M. K., Kostas, S. A., Driver, S. E. and Mello, C. C. 1998. Potent and specific genetic interference by double-stranded RNA in Caenorhabditis elegans. Nature 391: 806-811.

10. Ge, Q., McManus, M. T., Nguyen, T., Shen, C. H., Sharp, P. A., Eisen, H. N. and Chen, J. 2003. RNA interference of influenza virus production by directly targeting mRNA for degradation and indirectly inhibiting all viral RNA transcription. Proc. Natl. Acad. Sci. U. S. A. 100: 2718-2723.

11. Gitlin, L., Karelsky, S. and Andino, R. 2002. Short interfering RNA confers intracellular antiviral immunity in human cells. Nature 418: 430-434.

12. Hartmann, K., Donath, A., Beer, B., Egberink, H. F., Horzinek, M. C., Lutz, H., Hoffmann-Fezer, G., Thum, I. and Thefeld, S. 1992. Use of two virustatica (AZT, PMEA) in the treatment of FIV and of FeLV seropositive cats with clinical symptoms. Vet. Immunol. Immunopathol. 35: 167-175.

13. Hoffmann-Fezer, G., Thum, J., Ackley, C., Herbold, M., Mysliwietz, J., Thefeld, S., Hartmann, K. and Kraft, W. 1992. Decline in CD4+ cell numbers in cats with naturally acquired feline immunodeficiency virus infection. J. Virol. 66: 1484 1488.

14. Hu, W. Y., Myers, C. P., Kilzer, J. M., Pfaff, S. L. and Bushman, F. D. 2002. Inhibition of retroviral pathogenesis by RNA interference. Curr. Biol. 12: 1301-1311.

15. Huitron-Resendiz, S., De Rozieres, S., Sanchez-Alavez, M., Buhler, B., Lin, Y. C., Lerner, D. L., Henriksen, N. W., Burudi, M., Fox, H. S., Torbett, B. E., Henriksen, S. and Elder, J. H. 2004. Resolution and prevention of feline immunodeficiency virus-induced neurological deficits by treatment with the protease inhibitor TL-3. J. Virol. 78: 4525-4532.

16. Ikeda, Y., Tomonaga, K., Kawaguchi, Y., Kohmoto, M., Inoshima, Y., Tohya, Y., Miyazawa, T., Kai, C. and Mikami, T. 1996. Feline immunodeficiency virus can infect a human cell line (MOLT-4) but establishes a state of latency in the cells. J. Gen. Virol. 77: 1623-1630.

17. Jacque, J. M., Triques, K. and Stevenson, M. 2002. Modulation of HIV-1 replication by RNA interference. Nature 418: 435438.

18. Jiang, M. and Milner, J. 2002. Selective silencing of viral gene expression in HPV-positive human cervical carcinoma cells treated with siRNA, a primer of RNA interference. Oncogene 21: 6041-6048.

19. Lee, N. S., Dohjima, T., Bauer, G., Li, H., Li, M. J., Ehsani, A., Salvaterra, P. and Rossi, J. 2002. Expression of small interfering RNAs targeted against HIV-1 rev transcripts in human cells. Nat. Biotechnol. 20: 500-505.

20. Nishitsuji, H., Ikeda, T., Miyoshi, H., Ohashi, T., Kannagi, M. and Masuda, T. 2004. Expression of small hairpin RNA by lentivirus-based vector confers efficient and stable gene-suppression of HIV-1 on human cells including primary non-dividing cells. Microbes. Infect. 6: 76-85. 
21. Novina, C. D., Murray, M. F., Dykxhoorn, D. M., Beresford, P. J., Riess, J., Lee, S. K., Collman, R. G., Lieberman, J., Shankar, P. and Sharp, P. A. 2002. siRNA-directed inhibition of HIV-1 infection. Nat. Med. 8: 681-686.

22. Okuda, M., Minehata, K., Setoguchi, A., Cho, K. W., Nakamura, N., Nishigaki, K., Watari, T., Cevario, S., O'Brien, S. J., Tsujimoto, H. and Hasegawa, A. 1997. Cloning and chromosome mapping of the feline genes p21WAF1 and p27Kip1. Gene 198: 141-147.

23. Olmsted, R. A., Hirsch, V. M., Purcell, R. H. and Johnson, P. R. 1989. Nucleotide sequence analysis of feline immunodeficiency virus: genome organization and relationship to other lentiviruses. Proc. Natl. Acad. Sci. U. S. A. 86: 8088-8092.

24. Paul, C. P., Good, P. D., Winer, I. and Engelke, D. R. 2002. Effective expression of small interfering RNA in human cells. Nat. Biotechnol. 20: 505-508.

25. Pedersen, N. C., Ho, E. W., Brown, M. L. and Yamamoto, J. K. 1987. Isolation of a T-lymphotropic virus from domestic cats with an immunodeficiency-like syndrome. Science 235: 790793.

26. Randall, G., Grakoui, A. and Rice, C. M. 2003. Clearance of replicating hepatitis $\mathrm{C}$ virus replicon RNAs in cell culture by small interfering RNAs. Proc. Natl. Acad. Sci. U. S. A. 100: 235-240.

27. Rigby, M. A., Holmes, E. C., Pistello, M., Mackay, A., Brown, A. J. and Neil, J. C. 1993. Evolution of structural proteins of feline immunodeficiency virus: molecular epidemiology and evidence of selection for change. J. Gen. Virol. 74: 425-436.

28. Sharp, P. A. 2001. RNA interference--2001. Genes Dev. 15: 485-490

29. Shlomai, A. and Shaul, Y. 2003. Inhibition of hepatitis B virus expression and replication by RNA interference. Hepatology 37: 764-770

30. Snyder, H. W., Jr., Hardy, W. D., Jr., Zuckerman, E. E. and Fleissner, E. 1978. Characterisation of a tumour-specific antigen on the surface of feline lymphosarcoma cells. Nature 275: 656-658.

31. Wang, Z., Ren, L., Zhao, X., Hung, T., Meng, A., Wang, J. and Chen, Y. G. 2004. Inhibition of severe acute respiratory syndrome virus replication by small interfering RNAs in mammalian cells. J. Virol. 78: 7523-7527.

32. Yamamoto, J. K., Ackley, C. D., Zochlinski, H., Louie, H., Pembroke, E., Torten, M., Hansen, H., Munn, R. and Okuda, T. 1991. Development of IL-2-independent feline lymphoid cell lines chronically infected with feline immunodeficiency virus: importance for diagnostic reagents and vaccines. Intervirology 32: 361-375.

33. Zeng, Y. and Cullen, B. R. 2002. RNA interference in human cells is restricted to the cytoplasm. RNA 8: $855-860$.

34. Zheng, Z. M., Tang, S. and Tao, M. 2005. Development of resistance to RNAi in mammalian cells. Ann. New York Acad. Sci. 1058: 105-118. 